\title{
Not All Patients with Hemiplegia Need Alteplase: A Case of Hemiplegic Migraine
}

\author{
Sumeet Yadav ${ }^{1}$, Michelle Talukder², Jacky Duong ${ }^{1}$ \\ ${ }^{1}$ St. Joseph Mercy Oakland Hospital, Pontiac, USA \\ ${ }^{2}$ Ross University School of Medicine, Bridgetown, Barbados
}

Received: $17 / 02 / 2020$

Accepted: $18 / 02 / 2020$

Published: 02/04/2020

How to cite this article: Yadav S, Talukder M, Duong J. Not all patients with hemiplegia need alteplase: a case of hemiplegic migraine. EJCRIM 2020;7: doi:10.12890/2020_001585.

Conflicts of Interests: The Authors declare that there are no competing interests.

This article is licensed under a Commons Attribution Non-Commercial 4.0 License

\section{ABSTRACT}

Hemiplegic migraine (HM) is a rare type of migraine which presents with motor and sensory impairment like a cerebrovascular accident (CVA). We present a case of a 36-year-old female, with a prior CVA treated with alteplase, who presented to the emergency department with stroke-like symptoms with a duration of 1 hour. The NIH Stroke Scale score was 22 at presentation. The patient received alteplase under the supervision of a neurologist after head CT confirmed no bleed. Further work-up revealed no acute or remote ischaemia or infarction. This case demonstrates the importance of a thorough history, intercommunication between health systems and integrated Electronic Medical Records (EMR) for early diagnosis and management of HM.

\section{LEARNING POINTS}

- Hemiplegic migraine (HM) is difficult to diagnose and can mimic stroke, resulting in administration of dangerous medications such as thrombolytics/alteplase.

- A thorough history, intercommunication between health systems and integrated EMR are essential for early diagnosis and management of HM.

\section{KEYWORDS}

Hemiplegic migraine, alteplase, CVA, stroke

\section{INTRODUCTION}

Migraine can present with an aura. Auras are unequivocally localizable to the cerebral cortex or brain stem and include visual disturbances, sensory loss, dysphagia and motor involvement. A hemiplegic migraine (HM) is a rare entity in which the migraine presents with a motor aura. A motor aura includes an abnormality of movement such as hemiplegia and hemiparesis (80-99\%), ataxia and nystagmus (30-79\%), dysphagia (5-29\%) and some individuals will have visual changes (the percentage is unknown) ${ }^{[1]}$. Motor involvement including weakness mimics the symptom complex of stroke, and hence, HM could be misdiagnosed and treated as an ischaemic stroke. Diagnosis requires a careful patient history and exclusion of potential causes of symptomatic attacks.

\section{CASE DESCRIPTION}

A 36-year-old female, with a prior cerebrovascular accident (CVA), presented to the emergency department with a 1-hour episode of acute right facial droop, slurred speech and right-sided hemiparesis. Preceding this episode, she complained of light sensitivity and headache. The NIH Stroke Scale score was 22 at presentation. Head CT confirmed no bleed. The patient was given alteplase under the supervision of a neurologist. Further work-up for a CVA with brain MRI and a cerebral angiogram showed no acute or remote ischaemia or infarction. Cardiac 
and autoimmune disease work-ups were negative. The patient had no identifiable risk factors for a CVA and she recovered completely within 3 days. Detailed history-taking revealed that the patient was treated with alteplase 3 times for the same presentation from different hospitals in the last year. She was diagnosed with migraine and was prescribed topiramate; however, she failed to follow up due to financial reasons.

\section{DISCUSSION}

HM always presents clinically with motor involvement. There are similar provoking factors to those seen in patients with typical migraine, such as stress, exercise, bright lights, intense emotional influences and sleeping too much or too little. Based on family history, HM can be divided into sporadic HM (SHM) or familial HM (FHM). FHM often occurs earlier than typical migraine, typically in the first or second decade of life; however, the attack frequency tends to decrease with age ${ }^{[2]}$. In our case, the patient was more likely to have SHM because of the late onset of symptoms and she had no family history of HM.

The diagnostic criteria for $\mathrm{HM}$ are based on the International Classification of Headache Disorders, 3rd edition and are as follows ${ }^{[3]}$ :

1. Recurrent attacks, lasting minutes, of unilateral fully reversible visual, sensory or other central nervous system symptoms that usually develop gradually and are usually followed by headache and associated migraine symptoms.

2. Fully reversible motor weakness.

3. Fully reversible visual, sensory and/or speech/language symptoms.

4. FHM if at least 1 first-degree relative (that is, parent, sibling and/or offspring) or second-degree relative has identical attacks.

5. SHM if no first- or second-degree relative meets criteria for HM.

Many genes have been implicated in $\mathrm{HM}$ and are transmitted in an autosomal dominant pattern with variable penetrance. The genes involved in FHM are CACNA1A (FHM1), ATP1A2 (FHM2) and SCN1A (FHM3). 10-20\% of patients with SHM can have a gene mutation common to FHM, such as ATP1A2 (FHM2). The correlation between the genes associated with SHM and FHM is yet to be studied. Although genetic testing is available, it is not commonly used to confirm the diagnosis. A Danish population-based study carried out in 2007 showed that only $14 \%$ of patients with FHM had mutations in the genes listed above ${ }^{[4]}$. Further genetic characterization is yet to be conducted.

Genetic testing could aid with treatment in some cases. Each of these genes is associated with various presentations. FHM1 genes have been associated with cerebellar signs such as nystagmus and ataxia. FHM2 are associated with seizures. More recent studies have shown that mutations in PRRT2 genes that are known to be associated with benign familial infantile epilepsy and familial infantile convulsions with paroxysmal choreoathetosis are also associated with FHM. Hence, patients with FHM2 and PRRT2 mutations need seizure prophylaxis in addition to migraine prophylaxis ${ }^{[3,5]}$.

Management of HM is similar to the management of other types of migraine with aura. Medications such as flunarizine, lamotrigine, verapamil, acetazolamide, amitriptyline, topiramate and valproic acid are the mainstay of treatment. Clinical trials have not been conducted for prophylactic medication because of the rarity of the disease. However, prophylactic medication can be considered when the HM occurs more than twice per month or when it impairs the individual's quality of life ${ }^{[5]}$. Our patient was treated with topiramate when she was initially diagnosed with HM. However, she was not able to continue the treatment due to financial reasons, leading to repetitive attacks.

\section{CONCLUSIONS}

HM presents with neurological deficits mimicking an acute CVA, and hence, makes it a diagnostic challenge. Due to the impetus to treat patients with stroke symptoms and the code stroke protocol in many medical centres to reverse function loss, the patient may receive highly dangerous medications such as alteplase. When patients are using multiple medical centres that are not related, mistakes are likely to be repeated, as in our case. Hence, a careful history, detailed physical examination and assessment of risk factors for a CVA should be considered prior to initiation of stroke protocol and the administering of alteplase, especially in an ER setting. HM should be included in the differential diagnosis for a young patient without risk factors for a CVA, especially when the patient has repeat symptoms and episodes in a short period of time, such as our patient. Our patient presented with headache aura before progressing to sequential symptoms seen in $\mathrm{HM}$, and her symptoms completely resolved in 3 days, which is consistent with HM. Symptoms of a TIA or CVA have a sudden onset without aura and usually resolve or improve in 24 hours after receiving thrombolytic therapy. This case report represents an important clue for physicians to consider prior to making a diagnosis of an acute CVA and administering thrombolytic therapy. 


\section{REFERENCES}

1. The Genetic and Rare Diseases Information Centre (National Institutes of Health) [Internet]. Hemiplegic migraine [2014]. Available from: https://rarediseases.info.nih.gov/ diseases/10768/hemiplegic-migraine\#ref_3605 (accessed 16 February 2020).

2. Pelzer N, de Vries B, Kamphorst JT, Vijfhuizen LS, Ferrari MD, Haan J, et al. PRRT2 and hemiplegic migraine: a complex association. Neurology 2014;83:288-290.

3. Headache Classification Committee of the International Headache Society (IHS). The International Classification of Headache Disorders, 3rd edition (beta version). Cephalalgia 2013;33:629-808.

4. Thomsen LL, Kirchmann M, Bjornsson A, Stefansson H, Jensen RM, Fasquel AC, et al. The genetic spectrum of a population-based sample of familial hemiplegic migraine. Brain 2007;130:346-356.

5. Pelzer N, Stam AH, Haan J, Ferrari MD, Terwindt GM. Familial and sporadic hemiplegic migraine: diagnosis and treatment. Curr Treat Options Neurol 2013;15:13-27. 